\title{
Aerodynamic Models and Wind Tunnel for Straight-bladed Vertical Axis Wind Turbines
}

\author{
Qing'an LI*1, Takao MAEDA ${ }^{2}$, Yasunari KAMADA ${ }^{3}$, Junsuke MURATA ${ }^{4}$, \\ Kazuma FURUKAWA ${ }^{5}$ and Masayuki YAMAMOTO ${ }^{6}$ \\ ${ }^{1-4}$ (Division of System Engineering, Mie University,) \\ li@fel.mach.mie-u.ac.jp, maeda. Kamada,murata@mach.mie-u.ac.jp ) \\ 5, 6 (Graduate School of Engineering, Mie University) \\ Furukawa,yamamoto @fel.mach.mie-u.ac.jp)
}

\begin{abstract}
This paper has attempted to compile the main aerodynamic models that have been used for performance prediction and design of straight-bladed vertical axis wind turbine. Firstly, momentum models (specified as Rotor Blade Model and Streamtube Model) are basically based on calculation of flow velocity through turbine by equating the streamwise aerodynamic force on the blades with the rate of change of momentum of airflow, which is equal to the overall change in velocity times the mass flow rate. And then, according to this theory, Laser Doppler Velocimeter (LDV) was used to investigate two dimensional unsteady flow around Vertical Axis Wind Turbine at three different low tip speed ratios in wind tunnel. In order to clear the characteristics of power coefficient curve, the pressure distribution on the surface of rotor blade were also measured during rotation. Comparing the results, it is concluded that the power coefficient which is calculated from momentum models quantitatively agrees well with the experimental data, except when the blade is at a high tip speed ratio.
\end{abstract}

\section{KEYWORDS: - Wind Turbine, Momentum Model, Straight-bladed VAWT, Pressure Distribution, LDV}

\section{INTRODUCTION}

The capacity of wind power generation has increased rapidly in recent years. There has been growing interest in the development of small wind turbines with rotors only several meters in diameter for various applications such as street lights, environmental education, emergency power systems, and sources of distributed generation. According to the direction of rotor axis, wind turbines are classified into two categories: Horizontal Axis Wind Turbine (HAWT) and Vertical Axis Wind Turbine (VAWT) [1]. The main advantage of VAWT is that no yaw mechanisms are required, thus simplifying the design configurations significantly. Blades of straight-bladed VAWT may be of uniform section and untwisted, making them relatively easy to fabricate or extrude, unlike the blades of HAWT, which should be twisted and tapered for optimum performance. Furthermore, it reduces the transmission losses due to proximity to the demand center. So there is an urgent demand of this type of high efficiency with low cost in urban areas. Therefore, the design research on straightbladed VAWT became one of the hot spots of recent wind power technology development [2]. However, compared with the HAWT, there exists great prospect in developing VAWT in urban areas due to late start and immature technology.For predicting wind turbine performance, there exist many different methods. One of the most successful methods is the classical Blade Element Momentum theory (BEM) which was first introduced by Burton [3] to predict aerodynamic loading and power. The theory is based on one-dimensional momentum theory in which forces are distributed continuously in the azimuth direction.Based on the above principles, previously, performance prediction of VAWT has always been carried at measuring loads during rotation. However, the effect of the rotary shaft and steady from such tests cannot be considered. So, in this paper, effective test methods are introduced to clear these uncertainties concerning two of the aforementioned aspects: LDV (Velocity distribution) and multi-point pressure devices (Pressure distribution).The flow field characteristics were obtained through measuring the wind velocity in the flow field by LDV in the wind tunnel experiment. The aim of this test is to provide power coefficient which is lost from wind. In order to verify power coefficient which is obtained by wind turbine, accordance with multi-point pressure devices, the pressure on the rotor blade surface is measured. 


\section{NOTATIONS}
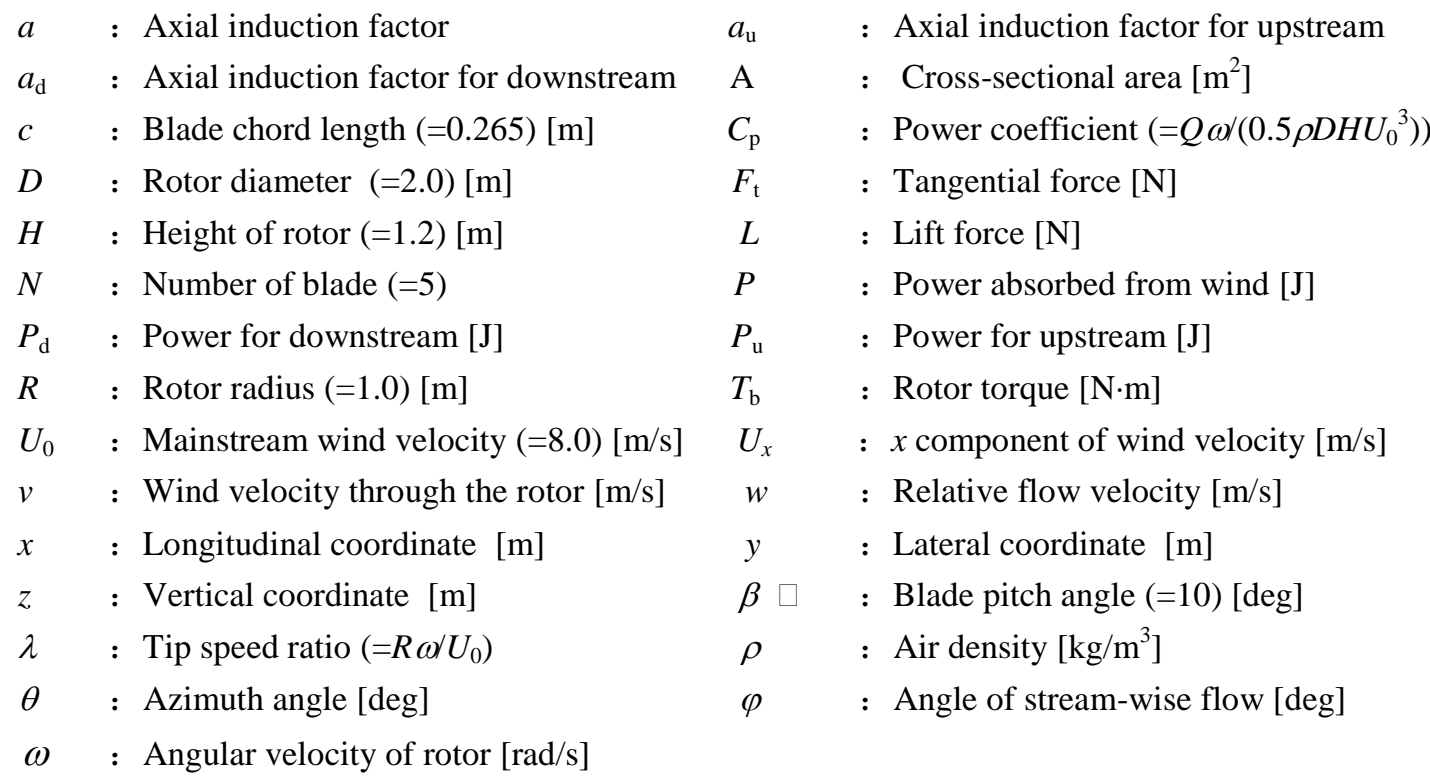

\section{MOMENTUM THEORY}

The methodology described in this paper has been developed with emphasis on two peculiarities of cross flow idealized wind turbines: the complicated flow surrounding vertical turbines and the sensitive dependency on various aerodynamic parameters. According to literature survey, the most widely studied and best validated models can be broadly classified into two categories-Rotor blade model and Streamtube model. These models are basically based on calculation of flow velocity and power through turbine by equating the streamwise aerodynamic force on blades with the rate of change of momentum of air, which is equal to the overall change in velocity times the mass flow rate [4]. The force is also equal to the average pressure difference across the rotor.

\subsection{Rotor blade model}

Rotor blade model refers to an analysis of velocities and forces at a section of the blade, as a function of blade geometry. Further, it provides very satisfactory results provided that good airfoil data are available for the lift and drag.

\subsubsection{Variation of angle of attack and relative flow velocity}

Fig. 1 shows the main velocities and forces relative to the blade chord line. From this figure one can observe that the flow is considered to occur in the axial direction. Referring to Fig. 1, the angle of attack $(\alpha)$ can be expressed as,

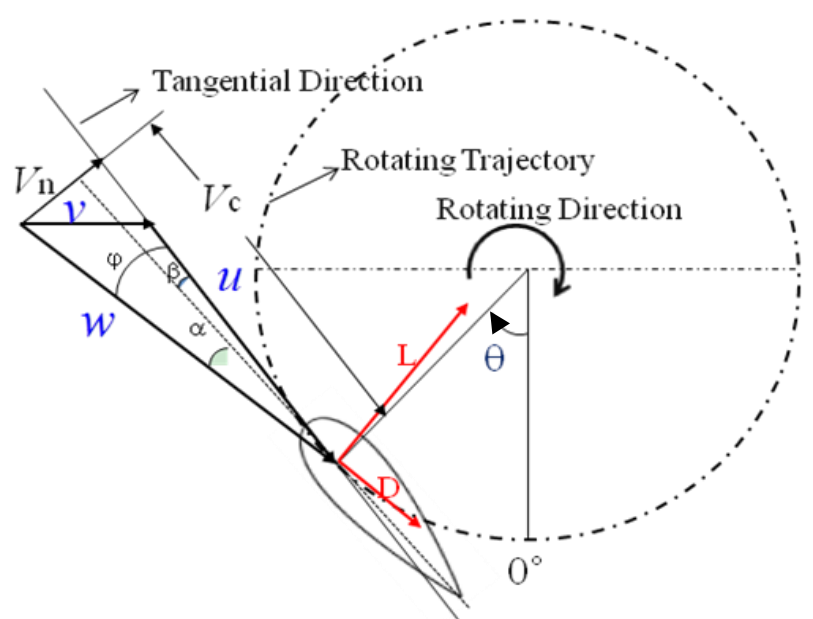

Fig.1 Blade Element Velocities and Forces Geometrical of SB-VAWT Blade 


$$
\alpha=\arctan \left[\frac{\sin \theta}{\left(\omega R / U_{0}\right) /\left(v / U_{0}\right)+\cos \theta}\right]-\beta
$$

where $v$ is the wind velocity through the rotor, $\omega$ is the rotational frequency of the turbine, $R$ is the radius of the rotor, and $\theta$ is the azimuth angle. $U_{0}$ is the mainstream wind velocity, and $\beta$ is the blade pitch angle.

The relative flow velocity $(w)$ can be obtained as,

$$
\frac{w}{U_{0}}=\frac{w}{v} \cdot \frac{v}{U_{0}}=\frac{v}{U_{0}} \sqrt{\left[\left(\frac{R \omega}{U_{0}} / \frac{v}{U_{0}}\right)+\cos \theta\right]^{2}+\sin ^{2} \theta}
$$

\subsubsection{Variation of tangential and normal forces}

The directions of the lift and drag forces and their normal and tangential components are shown in Fig 2. The tangential force Ft is basically the difference between the tangential components of lift and drag forces. From these relations, the expression of $\mathrm{Ft}$ can be determined like this:

$$
F_{\mathrm{t}}(\theta)=L \sin \varphi-D \cos \varphi
$$

where, $L$ is the lift force, and $D$ is the drag force. As shown in Fig. 1, the angle of resultant stream-wise flow $\varphi$ is then given by

$$
\varphi=\alpha+\beta
$$

Since, the tangential force represented by Eqs.(3) is for any azimuth position, so, it is considered as a function of azimuth angle $\theta$. Torque $\left(T_{\mathrm{b}}\right)$ acting on one blade can be expressed as,

$$
T_{\mathrm{b}}=R \int_{0}^{2 \pi} F_{\mathrm{t}}(\theta) \mathrm{d} \theta
$$

The amount of power $(P)$ for the number of blades $(N)$ and the power for upstream $\left(P_{\mathrm{u}}\right)$ and downstream $\left(P_{\mathrm{d}}\right)$ that can be absorbed from wind by a wind turbine, respectively, obtained from the following expressions:

$$
\begin{aligned}
P & =P_{u}+P_{\mathrm{d}} \\
P_{u} & =\frac{N R \int_{0}^{\pi} F_{\mathrm{t}}(\theta) \mathrm{d} \theta}{(2 \pi / \omega)} \\
P_{\mathrm{d}} & =\frac{N R \int_{\pi}^{2 \pi} F_{\mathrm{t}}(\theta) \mathrm{d} \theta}{(2 \pi / \omega)}
\end{aligned}
$$

Wind turbine rotor performance is usually characterized by its power coefficient, $C_{\mathrm{p}}$ :

$$
C_{\mathrm{p}}=\frac{P}{0.5 \rho A U_{0}^{3}}=\frac{\text { Rotor power }}{\text { Power in the wind }}
$$

where $\rho$ is the density of the air and A is the swept area of the turbine. The power coefficient represents the aerodynamic efficiency of the wind turbine and is a function of the tip speed ratio, $\lambda$ which is defined as

$$
\lambda=\frac{R \omega}{U_{0}}
$$

\subsection{Single streamtube model}

Streamtube model refers to an analysis of accounts for the extraction of kinetic energy when the wind goes through an ideal wind turbine. The turbine is represented by an actuator disk upon which a distribution of

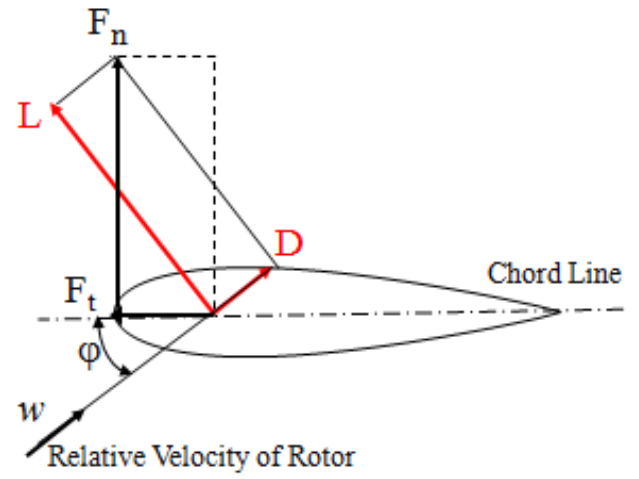

Fig.2 Forces Acting on Blade Geometrical Figure of SB-VAWT 
forces acts upon the mainstream at a rate defined by the period-averaged mechanical work that the rotor extracts from the fluid. These forces per unit area of power from wind in the whole operation process on the local flow field. In this model the entire turbine is assumed to be enclosed within a single streamtube as shown in Fig. 3. The general device that carries out this task is called an actuator disc. In actuator disc theory the induced velocity (rotor axial flow velocity) is assumed to be constant throughout the disc and is obtained by equating the streamwise drag with the change in axial momentum. Nevertheless, we can begin an analysis of the aerodynamic behaviour of wind turbines without any specific turbine design just by considering the energy extraction process [5].

The analysis assumes a control volume, in which the control volume boundaries are the surface of a stream tube and two cross-sections of the stream. Upstream of cross-sectional area $A_{0}$ is smaller than rotary area of wind turbine $A_{1}$ and $A_{1}$ is smaller than the disc downstream of cross-sectional area $A_{2}$. The mass of air which passes through a given cross-section of the stream-tube in a unit length of time is $\rho A U$, where $\rho$ is the air density, $A$ is the cross-sectional area and $U$ is the flow velocity. Now, according to Actuator Disk Theory, the mass flow rate must be the same everywhere along stream-tube and so

$$
\rho A_{0} U_{0}=\rho A_{1} U_{1}=\rho A_{2} U_{2}
$$

It is usual to consider that the actuator disc induces a velocity variation which must be superimposed on the mainstream velocity. If one defines the axial induction factor, $a$, as the fractional decrease in wind velocity between the mainstream and the rotor plane. At the disc, therefore, the section stream-wise velocity is

$$
U_{1}=U_{0}(1-a)
$$

The force causing this change of momentum comes entirely from the pressure difference across the actuator disc because the stream-tube is otherwise completely surrounded by air at atmospheric pressure, which gives zero net force. That force is equal and opposite to the thrust, $T$, which is the force of wind on the wind turbine. Therefore,

$$
T=A_{1} \Delta p=\rho A_{1} U_{1}\left(U_{0}-U_{2}\right)
$$

To obtain the pressure difference $\Delta p$ Bernoulli's equation is applied separately to the upstream and downstream sections of the stream tube.

Upstream, therefore, we have

$$
0.5 \rho U_{0}^{2}+p_{0}=0.5 \rho U_{1}^{2}+p_{1}^{+}
$$

Similarly, downstream,

$$
0.5 \rho U_{2}^{2}+p_{2}=0.5 \rho U_{1}^{2}+p_{1}^{-}
$$

where it is assumed that the far upstream and far downstream pressures are equal $(\mathrm{p} 0=\mathrm{p} 2)$, subtracting these equations we obtain

$$
\Delta p=p_{1}^{+}-p_{1}^{-}=0.5 \rho\left(U_{0}^{2}-U_{2}^{2}\right)
$$

Equation (11) and (12) then give

$$
0.5 \rho\left(U_{0}^{2}-U_{2}^{2}\right) A_{1}=\left(U_{0}-U_{2}\right) \rho A_{1} U_{0}(1-a)
$$

The force on the air becomes, from Equation (12)

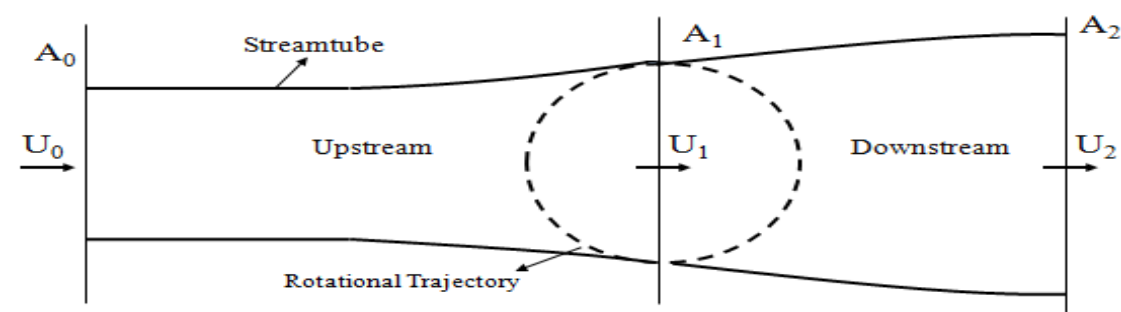

Fig.3 Schematic of Single Streamtube Model 


$$
T=A_{1} \Delta p=2 \rho A_{1} U_{0}^{2} a(1-a)
$$

Hence the power extraction from the air, $P$, is equal to the thrust times the velocity at the disc:

$$
P=T U_{1}=2 \rho A_{1} U_{0}^{3} a(1-a)^{2}
$$

Substituting for $P$ from Equation (19) into Equation （9）, the power coefficient, $C_{\mathrm{p}}$, is:

$$
C_{\mathrm{p}}=4 a(1-a)^{2}
$$

The maximum value of $C_{\mathrm{P}}$ occurs when $a=1 / 3$. Thus: $C_{\mathrm{P}}=16 / 27=0.5926$

This result indicates that, if an ideal rotor were designed and operated such that the wind velocity at the rotor were $2 / 3$ of the mainstream wind velocity, then it would be operating at the point of maximum power production. The maximum achievable value of the power coefficient is known as the Lanchester-Betz limit[6]. However, to date, no wind turbine has been designed that is capable of exceeding the Lanchester-Betz limit. The limit is caused not by any deficiency in design because, as yet in our discussion, we have no design. Here, because the stream-tube has to expand upstream of the actuator disc the cross-section of the tube where the air is at the full, mainstream velocity is smaller than the area of the disc.

\subsection{Double-multiple streamtube model}

In order to predict the power variations, in 1981, Paraschivoiu [7] introduced double multiple streamtube theory for the performance prediction of wind turbine. As shown in Fig. 4, in this model the swept volume of the turbine is divided into a series of adjacent and the calculation is done separately for the upstream and downstream half cycles of the turbine. The blade element and momentum theories are then employed for each streamtube. At each level of the rotor, the upstream and downstream induced velocities are obtained by using the principle of two actuator discs in tandem [8].The double-multiple streamtube model with constant and variable interference factors (induced velocity ratios), including secondary effects for wind rotor was examined. For both the upstream and downstream half cycles vertical variation of the induced velocity (like that in the multiple stream tube model) is considered while in the horizontal direction induced velocity is assumed to be constant (like that of a single streamtube model). For the upstream half-cycle, the wake velocity is represented by

$$
U_{2}=U_{0}\left(1-2 a_{u}\right)
$$

where $a_{\mathrm{u}}$ is the induced velocity factor $\left[a_{\mathrm{u}}=\left(U_{0^{-}} U_{2}\right) / U_{0}\right]$ for the upstream half-cycle. For the downstream halfcycle of the rotor, $U_{2}$ is the input velocity. The induced velocity for the downstream half-cycle is $U_{4}$ which can be written as

$$
U_{4}=U_{2}\left(1-2 a_{d}\right)
$$

where, $a_{\mathrm{d}}\left[a_{\mathrm{d}}=\left(U_{2}-U_{4}\right) / U_{2}\right]$ is the interference factor for the downstream half-cycle. The amount of power for upstream $\left(P_{S \mathrm{u}}\right)$ and downstream $\left(P_{S \mathrm{~d}}\right)$ that can be lost from wind, respectively, obtained from the following expressions:

$$
\begin{aligned}
& P_{S u}=0.5 \rho U_{0}^{2} A_{0} U_{0}-0.5 \rho U_{2}^{2} A_{2} U_{2} \\
& P_{S d}=0.5 \rho U_{2}^{2} A_{2} U_{2}-0.5 \rho U_{4}^{2} A_{4} U_{4}
\end{aligned}
$$

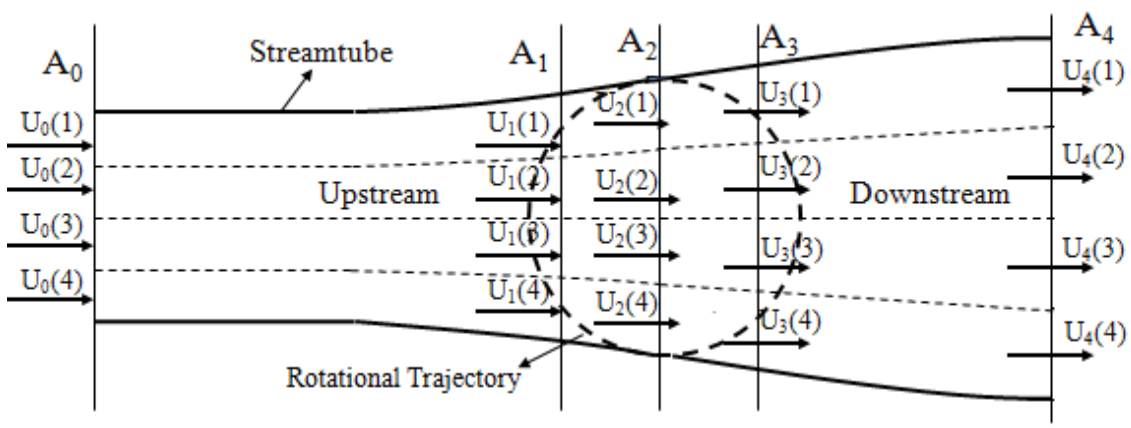

Fig.4 Schematic of Double-multiple Streamtube Model 


\section{EXPERIMENTAL APPARATUS AND METHOD}

\subsection{Experimental apparatus}

At present study, torque is measured by torque meter, and then, wind velocity around wind turbine in flow field is measured by LDV as shown in Fig.5; after that, in accordance with multi-point pressure devices as shown in Fig.6, the pressure on the blade surface is measured. Fig.7 shows the schematic diagram of the whole experimental apparatus. Experiments are carried out under the conditions that the mainstream velocity of $U_{0}=$ $8.0[\mathrm{~m} / \mathrm{s}]$, and blade pitch angle of $\beta=12\left[^{\circ}\right]$, for which wind turbine has the highest power output. LDV measures the wind velocity of the flow field at the tip speed ratios of $\lambda=0.95$ (low tip speed ratio), 1.39(optimum tip speed ratio) and 1.73 (high tip speed ratio). The coordinate system is defined for the measurements, in which the $x$-, the $y$ - and the $z$-axes are set in the mainstream, the lateral and the vertical directions, respectively. The origin is set at the center height location of wind turbine rotor. The focus of LDV is set at the center height of blade. Pitot tube is installed in 2070 of upstream from rotor axis, through which can determine mainstream velocity. Torque meter is installed in wind turbine axis of rotation, through which can determine rotor rotating speed and rotor torque.The measurements are carried out in an open test section of circular type wind tunnel in Mie University. The wind tunnel has an outlet diameter of $3600[\mathrm{~mm}]$ and its maximum wind speed reaches 30 $\mathrm{m} / \mathrm{s}$. Cross-sectional shape of the airfoil is the same as NACA0021 with five blades. The rotor has a diameter of $2000[\mathrm{~mm}]$, the blade height of $1200[\mathrm{~mm}]$ and blade chord length of $265[\mathrm{~mm}]$.

\subsection{Experimental methods}

The range of the experiment covers the entire rotation of the blade and almost the entire rotor area. As

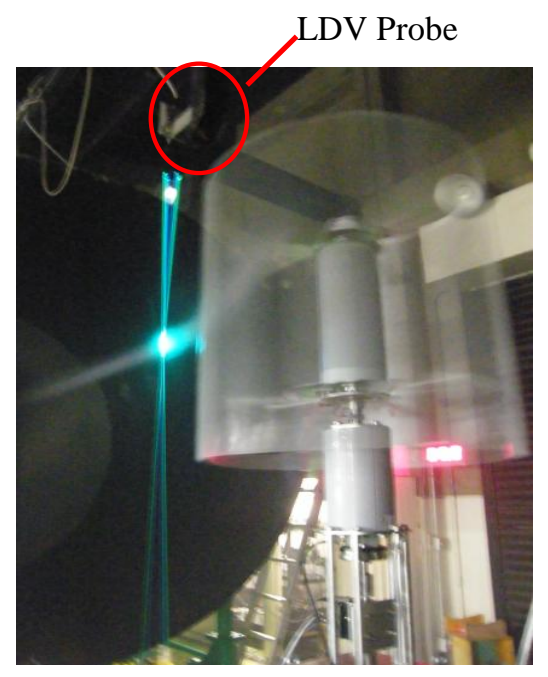

Fig. 5 LDV Measurement of VAWT Rotor

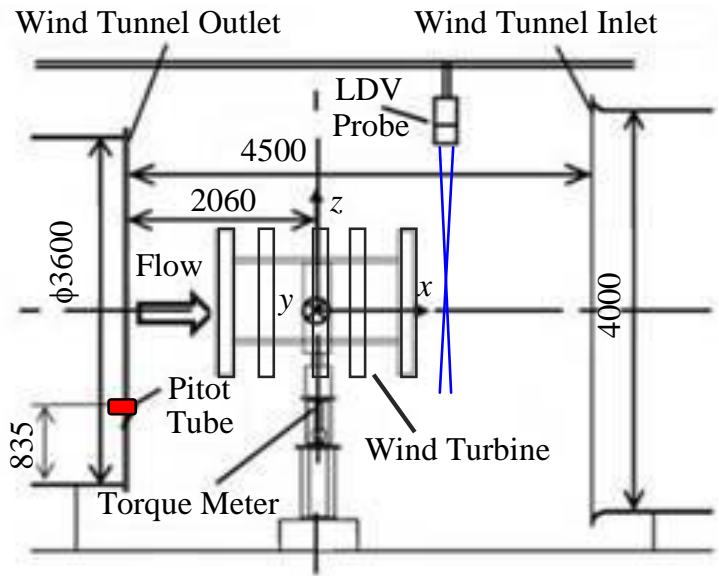

Fig. 7 Schematic Diagram of Experimental Apparatus

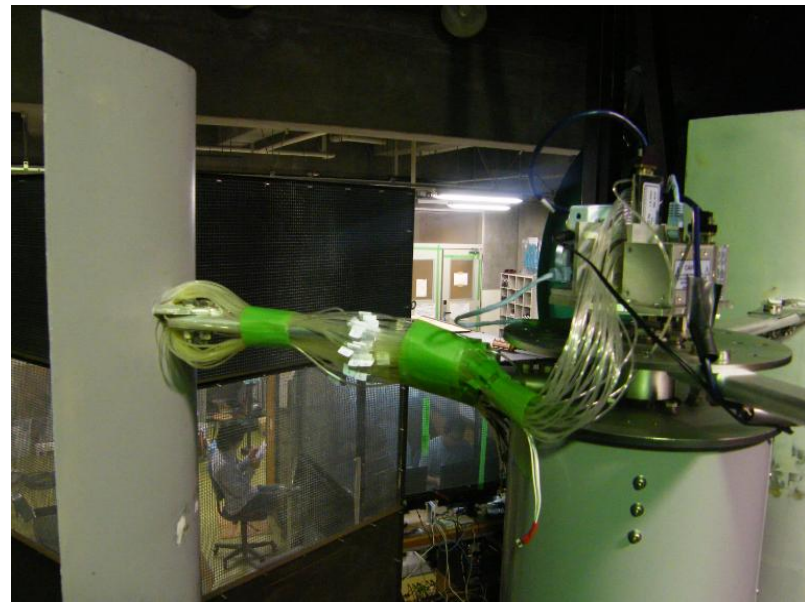

Fig.6 Photo of Multi-point Pressure Devices

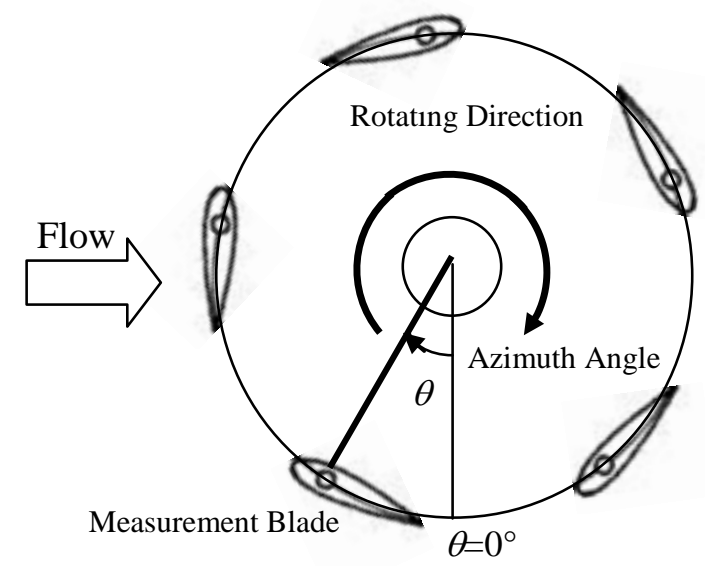

Fig. 8 Definition of Azimuth Angle 
shown in Fig.4, the measuring points of flow velocity are as follows: in the center height of airfoil direction $(z / R=0)$; in the mainstream flow direction there are eight cross-sections $(x / R=-1.8,-1.5,-1.0,-0.5,0,0.5,1.0,1.5$ and 2.0); in $\mathrm{y}$ axis direction, it is evenly spaced distribution at $\mathrm{y} / \mathrm{R}=0.2$ in the range of $-1.6 \leqslant y / R \leqslant 1.6$. The measuring plane is composed of a total of $17 \times 9=153$ measuring points. Although, the axis of rotation of the wind turbine is covered with a cylindrical cover of $216 \mathrm{~mm}$ diameter, so that in this range the measurement is not performed, the number of valid measuring points remained 152. Azimuth angle $\theta$, on the position where the travelling direction of blade matches the mainstream direction (the negative direction of $x$ ), is defined as $0^{\circ}$ as shown in Fig.8. Here, $\theta$ is specified as a positive direction in the direction of rotor rotation. As can be seen in Fig.9, $\beta$ is blade pitch angle, which is between chord line and track of rotor rotation. Wherein, direction in which the leading edge of airfoil outward is positive.Fig.10 shows research airfoil. In order to measure pressure distribution on the surface of rotor blade, in the span of airfoil center height, one of rotor blades is provided with a diameter of $0.4 \mathrm{~mm}$ pressure holes which the total quantity are 32. The red circles in Fig. 10 show the position of pressure measurement hole. The measurement hole can be perpendicularly drilled to blade surface, and arrangement of measurement hole is dense near the front edge with sharp pressure. The pressure on the surface of rotor blade is measured by pressure sensor which is installed in the upper rotation axis, and then transmitted through copper pipe and vacuum pipe by high-speed multipoint pressure measuring device. The detecting pressure signals in rotating system are transmitted to the stationary system through wireless LAN. The effects of centrifugal force and the dynamic response in the air of tubing are calibrated.

\section{RESULTS AND DISCUSSION}

\subsection{Flow flied and pressure distribution}

Fig.11 represents average velocity vector distribution at optimum tip speed ratio in flow flied. As can be seen, the velocity distribution is substantially uniform at upstream side $(x / R=-1.8)$ of wind turbines. Furthermore, approaching wind turbine in the upstream side of flow field at $x / R=-1.5$, the flow velocity decreases in the mainstream direction. The air flows outward to avoid the wind turbine as a result of the influence of the rotation of blades. According to Streamtube Model theory, it is confirmed that a wide low speed region appears from the wind turbine internal region to downstream, which is in good agreement with the experiment result. A conclusion section must be included and should indicate clearly the advantages, limitations, $\begin{array}{llllllllllll}\mathrm{a} & \mathrm{n} & \mathrm{d} & \mathrm{p} & \mathrm{o} & \mathrm{s} & \mathrm{s} & \mathrm{i} & \mathrm{b} & & 1 & \mathrm{e}\end{array}$

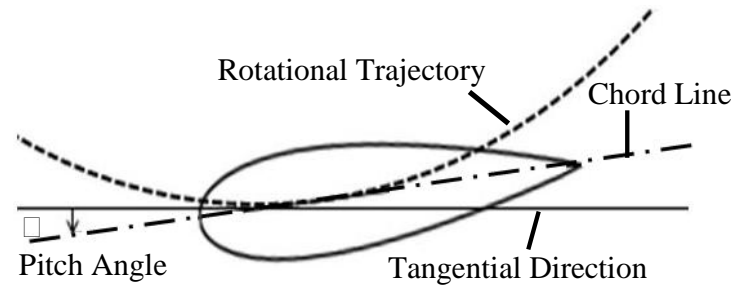

Fig. 9 Definition of Pitch Angle

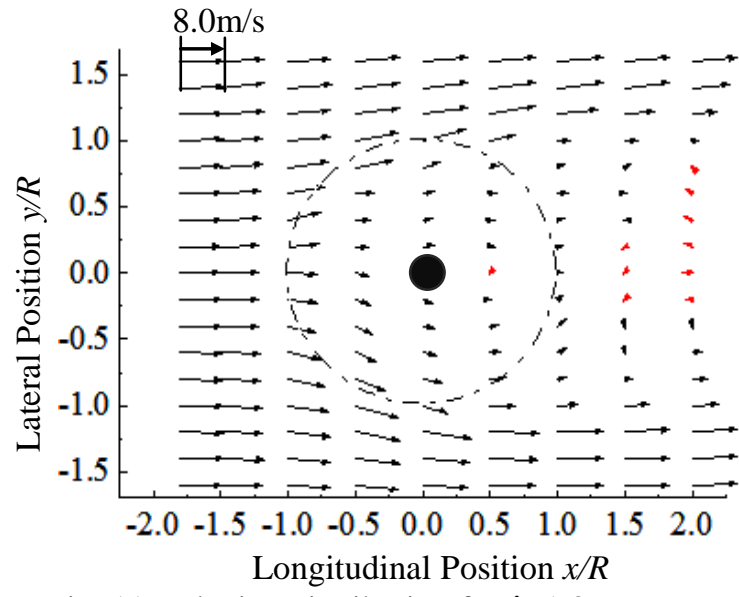

Fig. 11 Velocity Distribution for $\lambda=1.39$

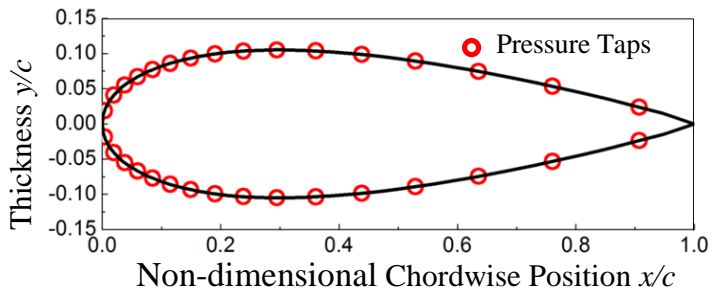

Fig.10 Airfoil

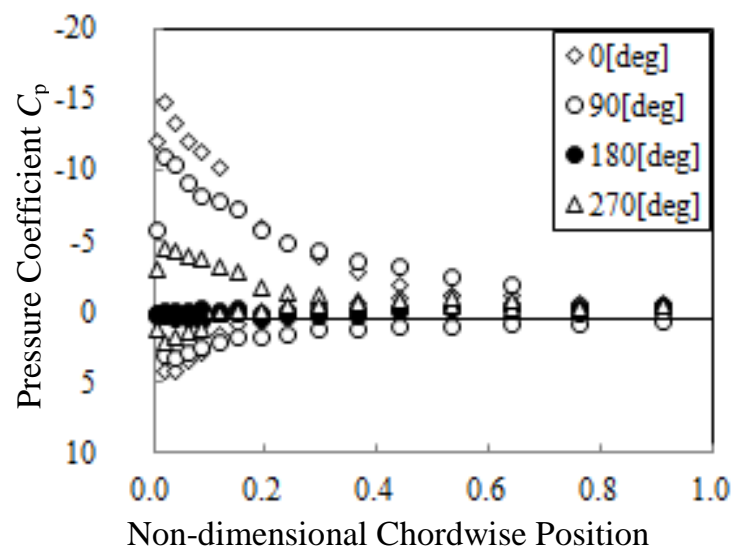

Fig. 12 Pressure Distribution on Blade $(\lambda=1.39)$ 
applications of the paper. Although a conclusion may review the main points of the paper, do not replicate the abstract as the conclusion. A conclusion might elaborate on the importance of the work or suggest applications and extensions.

At optimum tip wind speed, the pressure distribution on the rotor blade surface is respectively represented in Fig. 12 when $\theta=0\left[^{\circ}\right], 90\left[^{\circ}\right], 180\left[^{\circ}\right], 270\left[^{\circ}\right]$. According to the figure, we can know that when the rotor blade passes through the upstream side, the pressure of absolute becomes larger, and it will create a big fluid force at the same time. In contrast, when the wind turbine passes through the downstream side, the pressure of absolute will be reduced. It seems that compared with upstream side, the wind velocity of downstream side reduced (as shown in Fig.13) so that the relative angle of attack of rotor blade becomes smaller. Similar phenomenon appears in other tip speed ratios condition during rotor rotation.

\subsection{Power coefficient}

Fig.14 conveys the fluctuations of power coefficient $C_{\text {power }}$ which are obtained from pressure distribution on the rotor blade surface for one of blades during one rotation. This figure also shows the effects of three different tip speed ratios of $\lambda=0.95,1.39$ and 1.73 on the power coefficient of wind turbine. The vertical and horizontal axes represent the power coefficient $C_{\text {power }}$ and the azimuth angle $\theta$ of wind turbine.As shown in Fig.14, for low tip wind speed $\lambda=0.95$, at the downstream side, negative power coefficient is mainly generated. Positive power coefficient reaches its maximum at $\theta \approx 90^{\circ}$. However, for optimum and high tip wind speeds, at the downstream side, positive power coefficient are mainly generated. The power coefficients vary slightly at optimum and high tip wind speeds in the whole rotation. Along with the increase of tip speed ratio $\lambda$, there is an expanding tendency of the maximum power coefficient of a VAWT with one blade.

Meanwhile, from double-multiple streamtube model, the power difference for upstream $\left(P_{S \mathrm{u}}\right)$ and downstream $\left(P_{S \mathrm{~d}}\right)$ can be absorbed by Equation (23) - (24):

$$
\Delta P_{S}=P_{S u}-P_{S d}=0.5 \rho U_{2}^{3} A_{2} \times 4 a_{d}\left(1-a_{d}\right)
$$

As mentioned Actuator Disk theory, we can get $\Delta P_{\mathrm{s}}>0$. That is to say, it is very clear that the power absorbed from wind by a wind turbine mainly depends on upstream side. This is a very good agreement with the experiment result, as shown in Fig. 14.

The total power coefficient shown in Fig.15 is divided into the upstream and downstream power coefficients, as shown in Fig.15. The experimental values represent the power coefficient obtained from the pressure of blade surface by wind turbine, and the calculated values represent the power coefficient lost from the mainstream wind. According to Fig.15, it appears clearly that the calculated results are larger than experimental results. One of the important reasons is that wind velocity in vertical direction aroused by rotation of the rotor is not been considered. For purposes of the experimental values, for a tip speed ratio of $\lambda<1.28$, the downstream power coefficient becomes negative. For a tip speed ratio of $\lambda>1.28$, positive powers are generated in both the upstream and downstream region and the power coefficient is increasing with increasing tip speed ratio.

Fig.16 depicts the total power coefficients of calculated and experimental values. Calculated values include the power coefficients obtained from Single Streamtube Model (Single SM) and Double-multiple Streamtube Model (Double SM). Experimental values include the power coefficients obtained from torque meter and the pressure of blade surface by wind turbine. As shown in this figure, for calculated values, compared with Single SM, the power coefficient of Double SM is better agree with the experimental results from pressure distribution. However, compared with the results from pressure distribution, the difference is that the power coefficient of torque meter is gently decreasing with reducing the tip speed ratio from optimum one

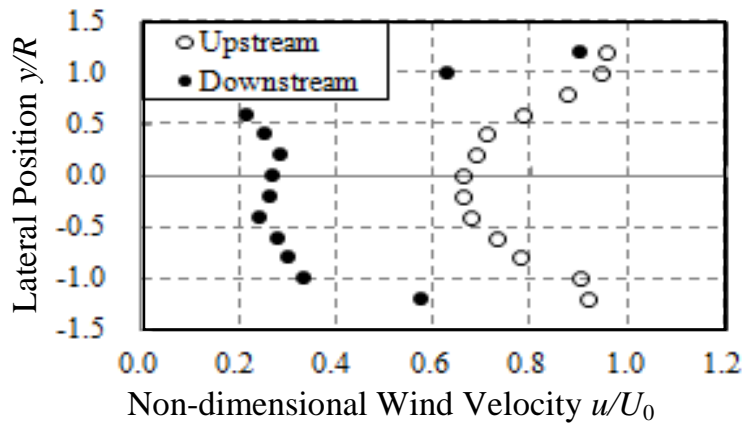

Fig.13 The Situation of Wind Velocity at $\lambda=1.39$ International organization of Scientific Research

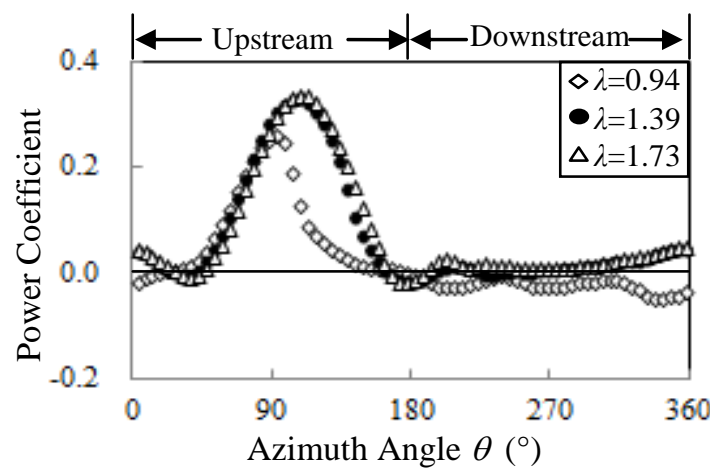

Fig.14 Power Coefficient Curve for One Blade 
$(\lambda=1.39)$. For experimental values, the power coefficient which is calculated from the pressure distribution becomes a higher value, especially in the high tip speed ratio. The power coefficient, which is based on the pressure distribution, has not considered the arm of the air resistance. Moreover, it also did not consider the great influence of blade tip vortex. The double-multiple streamtube model gives better correlation between the calculated and the experimental results of pressure distribution. However, the main drawback of these models is that they become invalid for large tip speed ratios and also for high rotor solidities because the momentum equations in these particular cases are inadequate [9].It should be noted that not all the models consider all the key components described above. The main effects lead to a decrease in the maximum achievable power coefficient as followed: Rotation of the wake behind the rotor; finite number of the blade and associated tip losses; non-zero aerodynamic; wind velocity at $\mathrm{z}$ axis aroused by rotation of the rotor [10].

\section{CONCLUSIONS}

This paper is aimed at clearing the power coefficient change of losing from wind and obtaining from a small type straight-bladed VAWT, using mathematical model and wind tunnel experiment. The following conclusions can be drawn:

(1) In flow field, a wide low wind velocity field will appear from the wind turbine internal region to downstream area. This is good agreement with the calculated result of Double-multiple Streamtube Model.

(2) About the pressure distribution on the rotor blade surface, when the rotor blade passes through the upstream side, the pressure of absolute becomes larger. In contrast, when the wind turbine passes through the downstream side, the pressure of absolute will be reduced.

(3) According to streamtube model, the power absorbed from wind by a wind turbine mainly depends on upstream side. This is a very good agreement with the experiment result of pressure distribution.

(4) For experimental values, the power coefficient which is calculated from the pressure distribution is good agreement with the calculated result, especially in the high tip speed ratio.

(5) For calculated values, compared with Single SM, the power coefficient of Double SM better agrees with the experimental results, especially in the experimental result of pressure distribution.

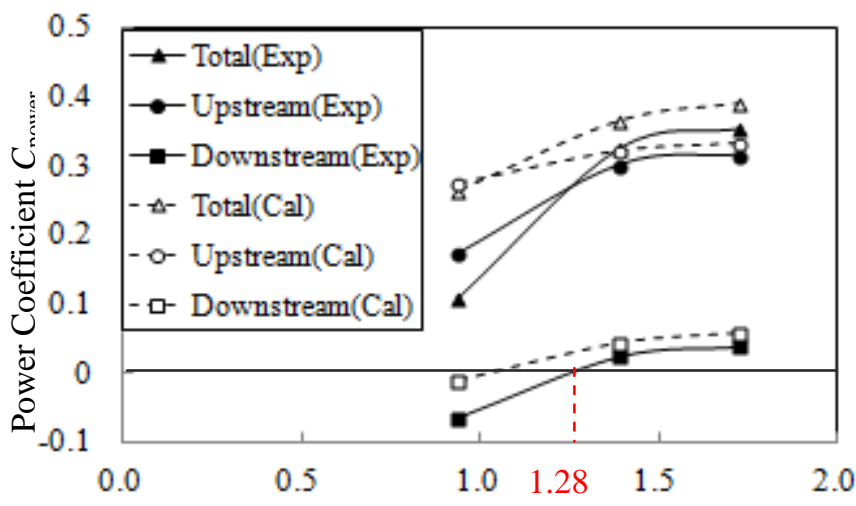

Tip Speed Ratio $\lambda$

Fig.15 Power Coefficients for Experiment and Calculated (Total, upstream, and downstream)

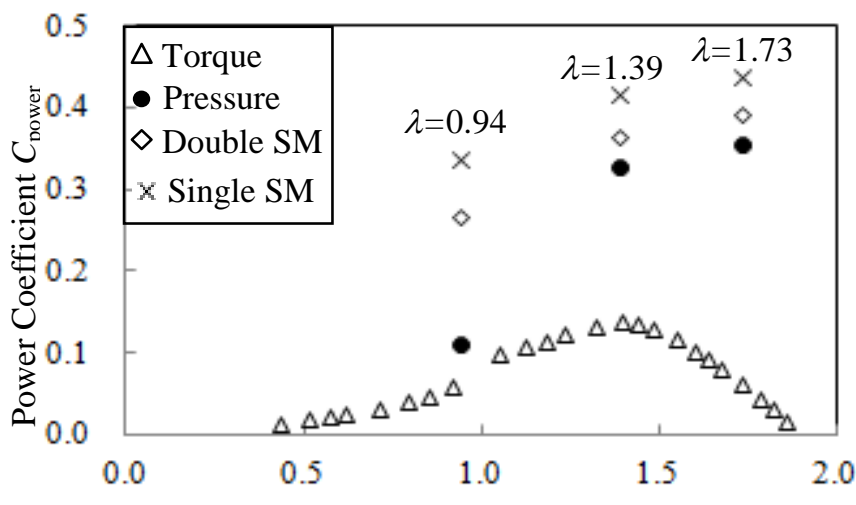

Tip Speed Ratio $\lambda$

Fig.16 Power Coefficient Curve for Torque, Pressure, Double SM and Single SM 


\section{ACKNOWLEDGEMENTS}

First and foremost, this work was supported by New Energy and Industrial Technology Development Organization (NEDO) in Japan. Last but not least, my sincere appreciation also goes to my team members Toshiaki KAWABATA and Naohiro MARUYAMA, for their helpful assistance during the experiment.

\section{REFERENCES}

[1] F. Scheurich, T.M. Fletcher, and R.E. Brown, Simulating the Aerodynamic Performance and Wake Dynamics of a Vertical-Axis Wind Turbine, Journal of Wind Energy, Vol. 14, No. 2, 2011, 159-177.

[2] T. Maeda, Y. Kamada, J. Murata, Q.A. Li, T. Kawabata and T. Kogaki, Measurements of flow field and pressure distribution of straight-bladed vertical axis wind turbine, Proc. European Wind Energy Association Conference and Exhibition, Austria, 2013.

[3] J.F. Manwell, J.G. McGowan1, A.L. Rogers, Wind Energy Explained: Theory, Design and Application, Second Edition. John Wiley \& Sons, 2009.

[4] C.S. Ferreira, G.V Kuik, G.V Bussel, and F. Scarano, Visualization by PIV of dynamic stall on a vertical axis wind turbine, Exp. Fluids, 2009, 97-108.

[5] M. Islam et al, Aerodynamic models for Darrieus-type straight-bladed vertical axis wind turbines, Renewable and Sustainable Energy Reviews, 12, 2008, 1087-1109.

[6] T. Burton, D. Sharpe, N. Jenkins, E. Bossanyi, Wind Energy Handbook, John Wiley Sons, New York, 2012.

[7] I. Paraschivoiu, Double-multiple streamtube model for darrieus wind turbines. Second DOE/NASA wind turbines dynamics workshop, NASA CP-2186, Cleveland, OH, 1981, 19-25.

[8] A. Mizuno, A. Iida, A. Shibuya, K. Fukudome. Kawabata and T. Kogaki, Performance Prediction of Straight Wings Vertical Axis Wind Turbines (Evaluation of Performance Prediction by Comparison Between Actuator Disc Theory and Vortex Methods Simulation), Japan Society of Mechanical Engineers, Japan, 2004, 509-512.

[9] D. A. Spera, Wind Turbine Technology-Fundamental Concepts in Wind Turbine Engineering Second Edition, ASME, Three Park Avenue, New York, 2009.

[10] RB. Noll, ND. Ham, Analytical evaluation of the aerodynamic performance of a high-reliability vertical-axis wind turbine. Proceedings of AWEA national conference, USA, 1980. 